\title{
Preparation and Characterization of Levofloxacin-Loaded Nanofibers as Potential Wound Dressings
}

\author{
Noémi Pásztor, Emőke Rédai, Zoltán-István Szabó, Emese Sipos* \\ University of Medicine and Pharmacy of Tîrgu Mureș, Romania
}

\begin{abstract}
Objective: The study aimed at obtaining and characterizing levofloxacin-loaded, poly( $\varepsilon$-caprolactone) electrospun nanofiber formulations to be used as antibacterial wound dressings. Methods: Drug-loaded nanofibers were obtained by the electrospinning process and their morphology was determined using scanning electron microscopy. Structural analysis of the prepared nanofibers was carried out using differential scanning calorimetry and dissolution testing was performed in order to determine drug release. Results: Both nanofiberous formulations (containing $20 \%$ and $50 \% \mathrm{w} / \mathrm{w}$ levofloxacin) showed dimensions in the range of few hundred nanometers. Thermograms indicated that the formulation containing $20 \%$ levofloxacin was totally amorphized, showing a rapid release of the active, in 20 minutes. Conclusions: The poly( $\varepsilon$-caprolactone)-based electrospun nanofibers, containing levofloxacin presented suitable characteristics for obtaining potential antibacterial wound dressings.
\end{abstract}

Keywords: electrospinning, polymer, scanning electron microscopy, differential scanning calorimetry

Received 1 March 2017 / Accepted 26 April 2017

\section{Introduction}

Wound dressings can play a major role in the healing process by ensuring a mechanical protection (to prevent secondary infection) and adequate conditions for wound healing. Ideally, wound dressing need to provide a moist environment while absorbing the excessive wound exudates, ensure adequate oxygen supply by allowing gaseous exchange and confer low bacterial exposure by incorporating a broad-spectrum antimicrobial agent [1]. They should also be non-traumatic and should not adhere to the wound, thus at dressing change they will not damage granulating tissue. It is claimed that also the immediate care of skin wounds is important for prevention of microbial infection and trans-epidermal water loss [2]. While the passive dressings fulfil very few of the properties of an ideal dressing and have very limited use as primary dressings, the novel interactive dressings may stimulate activity in the healing cascade and speed up the healing process [3].

Nanofibrous meshes prepared from polymeric materials are interesting alternatives to currently available wound treatments. Being characterized by ultra-fine, fibrous structure, high-surface area and nanoporosity, these meshes are excellent contenders for wound-healing applications [4]. Electrospinning is a simple and widely used technique to produce fibers with diameters in the range of several micrometers down to the few hundreds few hundreds of nanometer range from either polymer solutions or melts $[4,5]$. In the last decade much attention has been devoted to this technique, not only for the wide range of fiberforming polymers that can be used, but also because it can

* Correspondence to: Emese Sipos

E-mail: sipos.emese@yahoo.com consistently produce fibers in the submicron size range and active agents can be easily integrated. The fibers have smaller pore size and higher specific surface area than the ones produced with conventional methods and have been successfully applied in different fields, such as filtration, protective clothing manufacture, nanocatalysis, optical electronics and biomedicine [6-8].

Drug delivery systems based on nanofibers are able to improve therapeutic efficacy, bioavailability, reduce toxicity, and enhance patient compliance by delivering drugs at a controlled rate over a period of time to the site of action [9].

The obtained electrospun nanofibrous scaffolds can be used as a carrier for both hydrophilic and hydrophobic agents, where the drug release can be finetuned by the composition, morphology of the scaffolds and porosity. In the field of wound dressings and tissue engineering different active agents were successfully incorporated in electrospun scaffolds based on biocompatible polymers such as poly(ethylene-co-vinyl alcohol), collagen, polyurethane, poly(vinyl-alcohol), collagen-poly(ethylene-oxid), poly(lactic-co-glycolic acid)(PLGA), polylactic-acid(PLA) and gelatine $[4,10,11]$.

Studies also suggest that polymer matrices may have broad applicability in controlled release technology owing to the simplicity of the electrospinning process and the wide variety of suitable polymer materials[12-14].

These nanofibrous materials should be durable, stress resistant, flexible and elastic in order to be employed as wound dressings. They also should be easy to apply and remove without incurring any trauma during dressing change. Poly(E-caprolactone) (PCL) based nanofibrous mats fulfil the abovementioned requirements and were 
successfully applied as wound dressing in numerous studies, showing similar or superior efficacy, when compared to standard therapy [15-17].

The model drug chosen for this research was levofloxacin, which is a water-soluble fluoroquinolone-type antibacterial agent, with broad-spectrum antimicrobial spectrum and enhanced efficacy against Gram-positive and atypical pathogens [18]. Levofloxacin is especially important because of its activity on strains that are resistant to other, widely applied antimicrobial agents. This additional activity is especially useful for developing novel wound dressings for severe bacterial infections of the skin [19].

The aim of the present study was to obtain and characterize levofloxacin-loaded nanofibrous matrices from PCL prepared by electrospinning. The drug release profiles, morphology and thermal properties of the scaffolds are presented herein. Results enable us to understand the effects of the applied polymer and active substance concentrations on the properties of potential wound dressing materials.

\section{Materials and Methods}

\section{Materials}

The model drug, Levofloxacin (Figure 1) was provided by Richter Gedeon Plc. (Budapest, Hungary).

Poly( $\varepsilon$-caprolactone) (PCL, $50 \mathrm{kDa}$, Figure 1), ethanol, dichloromethane, sodium hydroxide $(\mathrm{NaOH})$ and phosphate buffer solution (1M, $\mathrm{pH} 7.4)$ were purchased from Sigma-Aldrich.

\section{Preparation of PCL dispersions with levofloxacin}

As a first step, five different formulations were prepared in order to select a homogeneous PCL dispersion. The exact compositions of dispersions and their aspects are presented in Table I.

As only formulation F5 resulted in a homogenous composition, only this composition was further used for incorporating levofloxacin. The active substance was dissolved in this formulation, obtaining final concentrations of $20 \%$ and $50 \%$, respectively. All active substance-related procentual concentrations indicated in the present study are expressed as weight per weight (w/w \%).

\section{Electrospinning process}

The abovementioned formulations (F5 containing 20\% and $50 \%$ levofloxacin) were loaded into a $10 \mathrm{~mL}$ syringe, which was connected through a $30 \mathrm{~cm}$ long silicone tube to a $0.8 \mathrm{~mm}$ internal diameter spinneret at the end. The constant flow of the polymeric solution was provided by a SEP 10S Plus automatic syringe pump (Aitecs, Lithuania). The electrostatic spinner was equipped with a NT35 adjustable high-voltage DC power supply (MA2000, Unitronik, Hungary). A vertically-positioned, grounded collector, covered with aluminium foil was positioned at a distance of $30 \mathrm{~cm}$ from the spinneret. The flow rate was set at $10 \mathrm{ml} / \mathrm{h}$, while the applied voltage was $35 \mathrm{kV}$.

\section{Scanning electron microscopy (SEM)}

Morphology of the samples was investigated by a JEOL 6380LVa (JEOL, Tokyo, Japan) type scanning electron microscope. Each specimen was fixed by conductive double sided carbon adhesive tape and sputtered by gold (using JEOL 1200 instrument) in order to avoid electrostatic charging. The diameters of the fibers were determined with the aid of the software provided by the SEM analyzer.

Table I. Compositions of different PCL dispersions

\begin{tabular}{lcccc}
\hline Components & F1 & F2 & F3 & F5 \\
\hline PLC $(\mathrm{g})$ & 1.00 & 2.00 & 3.00 & 1.00 \\
Ethanol $(\mathrm{ml})$ & 8 & 8 & 8 & 8 \\
Dichloromethane $(\mathrm{ml})$ & - & - & -60 & 4 \\
Aspects & Inhomogeneous & Inhomogeneous & Inhomogeneous & Gelling \\
\hline
\end{tabular}
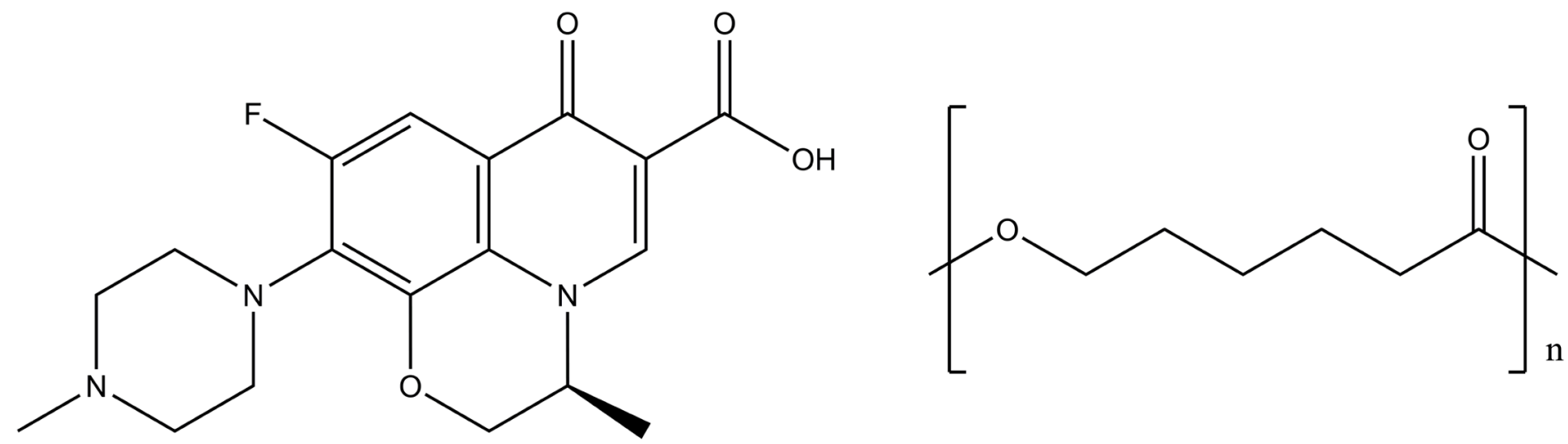

(A)

Fig. 1. Chemical structure of (A) levofloxacin and (B) PCL 


\section{Differential Scanning Calorimetry (DSC)}

The DSC studies were performed on a Perkin Elmer DSC 7 type device using nitrogen as carrier gas. The weight of the samples varied between 10 and $20 \mathrm{mg}$. The temperature was raised from $-5^{\circ} \mathrm{C}$ to $300^{\circ} \mathrm{C}$ with a rate of $10^{\circ} \mathrm{C} /$ min.

\section{In vitro drug dissolution measurement}

The dissolution studies were performed by a Pharmatest IDS 1000 dissolution apparatus equipped with rotating paddles (Pharmatest, Germany). Electrospun samples containing $20 \%$ and $50 \%$ active were placed in the dissolution vessel filled with $900 \mathrm{ml}$ phosphate buffer solutions with $\mathrm{pH}=7.4$ maintained at $37 \pm 0.5^{\circ} \mathrm{C}$ and stirred at $50 \mathrm{rpm}$. At the predetermined timepoints, $5 \mathrm{~mL}$ samples were withdrawn and analyzed using a Hewlett-Packard HP 8452A UV-VIS spectrophotometer (Palo Alto,USA) at $288 \mathrm{~nm}$. Concentrations were calculated based on a calibration curve (the polymer matrices had no detectable absorbance at this wavelength).

\section{Results and Discussion}

\section{Morphology of the electrospun fibers}

After preliminary optimization of the solution compositions, the morphology of the produced fibers were examined and compared. Figure 2 represents the SEM images of the PCL fibers without active substance and that of $20 \%$ and 50\% drug-loaded fibers. In all cases, the obtained fibers presented diameters in the micrometer to few hundreds of nanometer range, suitable for their intended use.

Differential Scanning Calorimetry (DSC) measurements

DSC curves preseted in Figure 3 confirm the crystallineamorphous transition of levofloxacin through fiber formation. The thermogram of levofloxacin presents a sharp melting endotherm at $235^{\circ} \mathrm{C}$, while the DSC curves of the nanofibrous material show an endothermic peak at 62 ${ }^{\circ} \mathrm{C}$, characteristic for PCL melting [20]. The sharp endotherm of melting disappears from the thermograms of 20 $\%$ drug-loaded nanofibers, while it is greatly reduced and shifted towards lower temperatures at $50 \%$ levofloxacin content. One possible explanation of the abovementioned phenomenon is the amorphisation of the active substance during the electrospinning process.

\section{Dissolution tests of the levofloxacin nanofibers}

Figure 4 represents the drug release profiles of nanofibers with $20 \%$ and $50 \%$ levofloxacin carried out in phosphate buffer $\mathrm{pH} 7.4$.

It can be observed that levofloxacin is totally released from the polymer matrix within 20 minutes and 4 minutes from the $20 \%$ and $50 \%$ levofloxacin-loaded nanofibers, respectively. At the end of the dissolution test, after the release of the active the PCL matrix remained visually unchanged, indicating that it could be a suitable wound dressing, which releases its active content, but does not deteriorate when comes into contact with liquids or body fluids.

\section{Conclusions}

This study showed that PCL can be a promising matrix for the preparation of antimicrobial wound dressing. Due

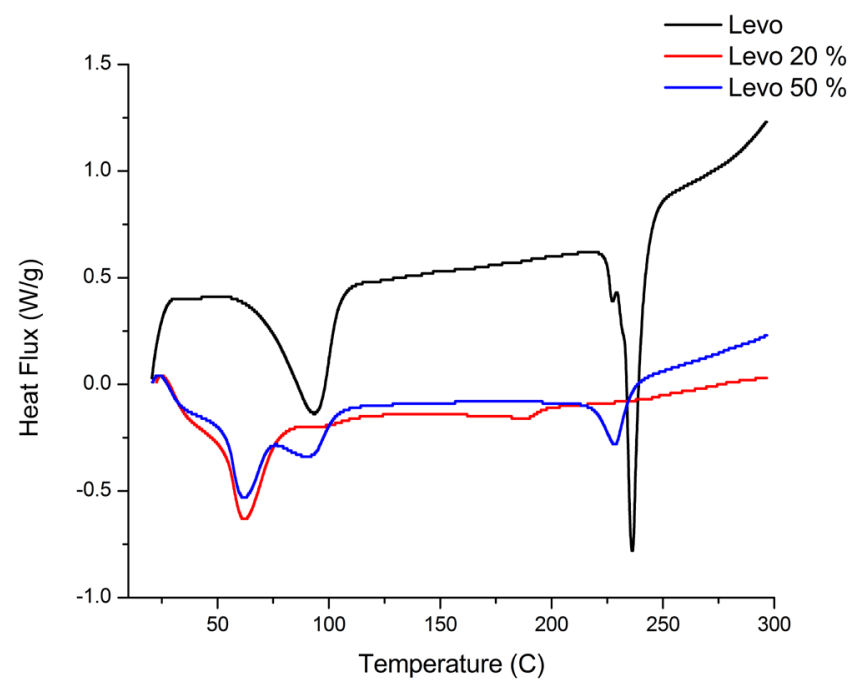

Fig. 3. DSC thermograms of levofloxacin (Levo, upper trace, blue), $50 \%$ levofloxacin-loaded nanofibers (Levo $50 \%$, middle trace, gray) and $20 \%$ levofloxacin-loaded nanofibers (Levo 20\%, lower trace, orange).

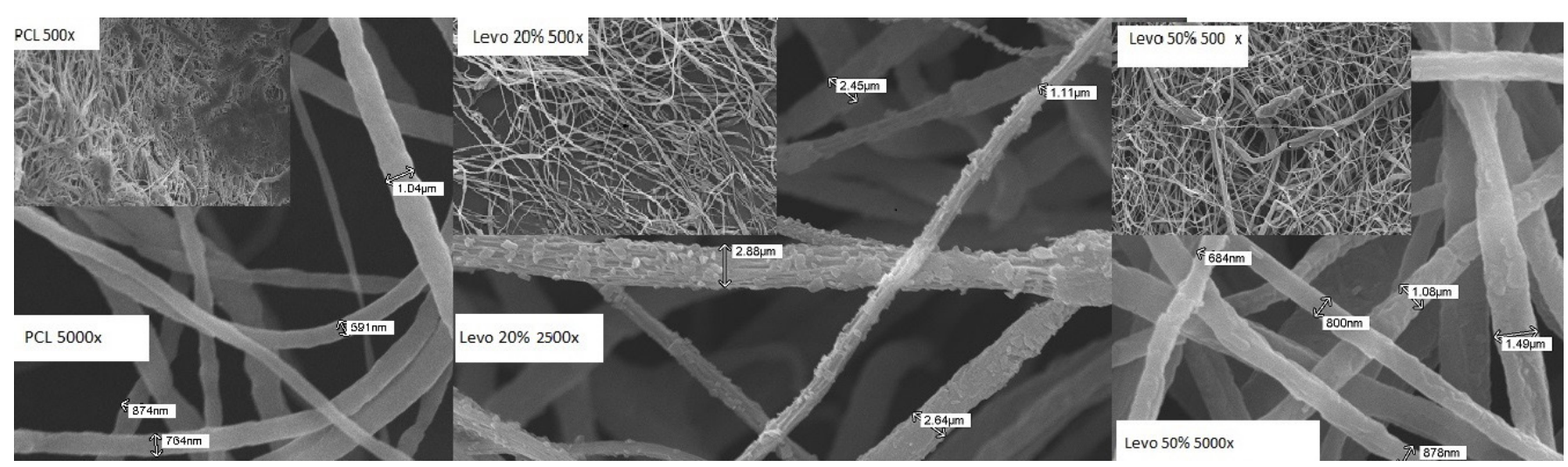

Fig. 2. SEM photographs of the prepared nanofibers using different magnifications: Nanofibers without active substance (PCL), $20 \%$ levofloxacin-loaded nanofibers (Levo $20 \%$ ) and $50 \%$ levofloxacin-loaded nanofibers (Levo $50 \%$ ) 


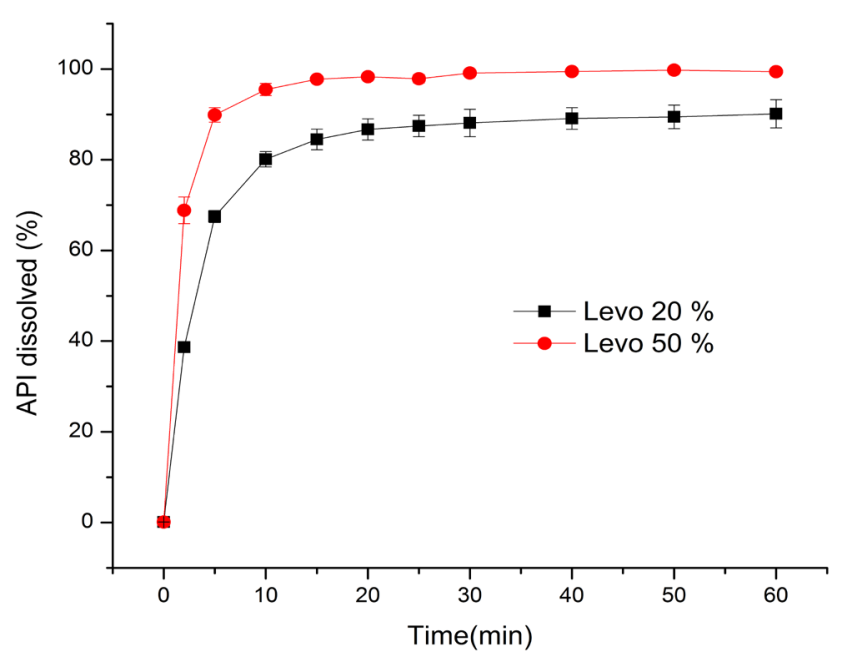

Fig. 4 .Comparative dissolution studies of $20 \%$ (Levo 20\%) and 50 $\%$ (Levo 50\%) levofloxacin-loaded nanofibers

to its stable and elastic structure, the polymer can be suitable for topical application of the manufactured dressings as it fully releases the active and does not degrade when it comes into contact with body fluids. DSC studies confirmed the crystalline-amorphous transition of the active substance during the electrospinning process. The formulated nanofibrous mats may be expected to deliver an attacking dose of levofloxacin, which could be very useful after surgery or in cases of severe topical infections.

\section{Conflict of interest}

None declared.

\section{Aknowledgements}

This study was supported by an internal research grant of the University of Medicine and Pharmacy of Tirgu Mures and SC Gedeon Richter Romania SA.

\section{References}

1. Boateng JS, Matthews KH, Stevens HNE, Eccleston GM - Wound healing dressings and drug delivery systems: a review. J Pharm Sci. 2008; 97: 2892-2923.

2. Sikareepaisan P, Ruktanonchai U, Supaphol P - Preparation and characterization of asiaticoside-loaded alginate films and their potential for use as effectual wound dressings. Carbohydr Polym. 2011; 83: 1457-
1469.

3. Queen D, Orsted H, Sanada H, Sussman G - A dressing history. Int Wound J. 2004; 1: 59-77.

4. Abrigo M, McArthur SL, Kingshott P - Electrospun nanofibers as dressings for chronic wound care: advances, challenges, and future prospects. Macromol Biosci. 2014; 14: 772-792.

5. Reneker DH, Yarin AL, Zussman E, Xu H - Electrospinning of nanofibers from polymer solutions and melts. Adv Appl Mech. 2007; 41, 43-346.

6. Subbiah T, Bhat GS, Tock RW, Parameswaran S, Ramkumar SS Electrospinning of nanofibers. J Appl Polym Sci. 2005; 96: 557-569.

7. Ramakrishna S, Fujihara K, Teo WE, Yong T, Ma Z, Ramaseshan R Electrospun nanofibers: solving global issues. Mater today. 2006; 9: 40-50.

8. Bhardwaj N, Kundu SC - Electrospinning: a fascinating fiber fabrication technique. Biotechnol Adv. 2010; 28: 325-347.

9. Kenawy ER, Abdel-Hay FI, El-Newehy MH, Wnek GE - Processing of polymer nanofibers through electrospinning as drug delivery systems. Mater Chem Phys. 2009; 113: 296-302.

10. Pham QP, Sharma U, Mikos AG - Electrospinning of polymeric nanofibers for tissue engineering applications: a review. Tissue Eng. 2006; 12: 1197-1211.

11. Supaphol $P$, Suwantong $O$, Sangsanoh $P$, Srinivasan $S$, Jayakumar $R$, Nair SV - Electrospinning of biocompatible polymers and their potentials in biomedical applications. Springer Berlin Heidelberg, 2011; pp. 213 239.

12. Thakur RA, Florek CA, Kohn J, Michniak BB - Electrospun nanofibrous polymeric scaffold with targeted drug release profiles for potential application as wound dressing. Int J Pharm. 2008; 364: 87-93.

13. Jannesari M, Varshosaz J, Morshed M, Zamani M - Composite poly (vinyl alcohol)/poly (vinyl acetate) electrospun nanofibrous mats as a novel wound dressing matrix for controlled release of drugs. Int $J$ Nanomedicine. 2011; 6: 993-1003.

14. Feng K, Sun H, Bradley MA, Dupler EJ, Giannobile WV, Ma PX - Novel antibacterial nanofibrous PLLA scaffolds. J. Control Release 2010; 146: 363-369.

15. Duan Y, Jia J, Wang S, Yan W, Jin L, Wang Z - Preparation of antimicrobial poly (??-caprolactone) electrospun nanofibers containing silver-loaded zirconium phosphate nanoparticles. J Appl Polym Sci. 2007; 106: 12081214.

16. Merrell JG, McLaughlin SW, Tie L, Laurencin CT, Chen AF, Nair LS Curcumin-loaded poly (?-caprolactone) nanofibres: Diabetic wound dressing with anti-oxidant and anti-inflammatory properties. Clin Exp Pharmacol Physiol. 2009; 36: 1149-1156.

17. Zahedi P, Karami Z, Rezaeian I, Jafari SH, Mahdaviani P, Abdolghaffari $\mathrm{AH}, \mathrm{Abdollahi} \mathrm{M}$ - Preparation and performance evaluation of tetracycline hydrochloride loaded wound dressing mats based on electrospun nanofibrous poly (lactic acid)/poly (?? -caprolactone) blends. J Appl Polym Sci. 2012; 124: 4174-4183.

18. Wimer SM, Schoonover L, Garrison MW - Levofloxacin: a therapeutic review. Clin. Ther. 1998; 20: 1049-1070.

19. Toncheva A, Spasova M, Paneva D, Manolova N, Rashkov I - Polylactide (PLA)-based electrospun fibrous materials containing ionic drugs as wound dressing materials: a review. Int J Polym Mater Polym Biomater. 2014; 63: 657-671.

20. Thomas V, Jose MV, Chowdhury S, Sullivan JF, Dean DR, Vohra YK Mechano-morphological studies of aligned nanofibrous scaffolds of polycaprolactone fabricated by electrospinning. J Biomater Sci Polym Ed. 2006; 17: 969-984. 